\title{
Body adiposity index in assessing the risk of type 2 diabetes mellitus development: the Baependi Heart Study
}

\author{
Camila Maciel de Oliveira ${ }^{1,2^{*}}$ C, Jessica Pavani ${ }^{3}$, José Eduardo Krieger ${ }^{1}$, Rafael de Oliveira Alvim ${ }^{4}$ \\ Carlos Alberto Mourão-Junior ${ }^{5}$ and Alexandre da Costa Pereira ${ }^{1}$
}

\begin{abstract}
Background: The association between diabetes and obesity is very well established. Faced with this, several anthropometric indices of adiposity are often involved in studies on diabetes. Our main goal in this paper is to evaluate the association between body adiposity index (BAI) and type 2 diabetes mellitus (T2DM) in a sample of the Brazilian population after 5-year follow-up.

Methods: The data used come from the Baependi Heart Study cohort, which consists of two periods: cycle 1 (20052006) and cycle 2 (2010-2013). Individuals of both sexes $(n=1121)$ were selected by excluding participants with type 2 diabetes mellitus at baseline or those that were lost to follow-up.

Results: The diabetic subjects showed higher systolic blood pressure, BAl, body mass index, waist circumference and fasting glucose levels. In addition, using mixed-effects logistic regression, we found that the elevation of a single unit of BAl represented an increase of $8.4 \%$ in the risk of a patient developing T2DM (OR $=1.084$ [95\% $\mathrm{Cl} 1.045-1.124]$ ).
\end{abstract}

Conclusions: Obesity is recognised as one of the most important risk factors for T2DM and BAI has proven to be a useful tool in estimating the risk of a patient developing T2DM in a Brazilian population.

Keywords: Baependi heart study cohort, Body adiposity index, Brazil, Type 2 diabetes mellitus

\section{Introduction}

Diabetes Mellitus is a multifactorial metabolic disease associated with various conditions, including genetic predisposition, physical inactivity and mainly obesity. In fact, obesity is the second most important risk factor for various diseases [1] and its association with diabetes is very well established $[2,3]$. As in the rest of the world, obesity has attracted attention in Brazil. More than half of the Brazilian population is overweight and almost $20 \%$ is obese [1]. The increasing incidence of obesity and increase in the incidence of type 2 diabetes (T2DM) has caused great concern about public policy.

\footnotetext{
*Correspondence: camilamacieloliveira@gmail.com

${ }^{2}$ Department of Integrative Medicine, Federal University of Paraná,

Curitiba, Brazil

Full list of author information is available at the end of the article
}

Many studies have been carried out in this area, however, many gaps still need to be bridged. Previous studies have commonly discussed the effectiveness of anthropometric indices of adiposity in association with some cardiovascular risk factors [4]. Of these, the simplest ones, such as body mass index (BMI) and waist circumference (WC), have been most widely explored by the scientific community, although their limitations are well understood as applied to the prediction of pathologies such as T2DM in different populations. As an alternative, other parameters have been proposed, such as the body adiposity index (BAI), which take account simple measures such as hip circumference and height. This parameter, developed by Bergman et al. [5], has proven to be effective when related to some metabolic diseases.

Comparisons between BAI and other adiposity indexes have been also explored [6, 7]. However, according to Alvim et al. [2], ethnic differences may influence the 
discriminatory power of BAI, as well as several other anthropometric indices, in assessing the risk of T2DM. In agreement with this reality, our main interest is to investigate the influence of BAI on T2DM in a sample of the Brazilian population, after 5-year follow-up.

\section{Methods}

The Baependi Heart Study cohort is a genetic epidemiological follow-up study of cardiovascular risk factors [8]. For the data collection process, individuals of both genders and aged 18-102 years were randomly considered, all from the municipality of Baependi. After recruitment, all relatives of the participants were invited to participate. Once selected, a physical examination was carried out and blood samples were collected. Currently, the cohort study consists of two periods: cycle 1 (2005-2006) and cycle 2 (2010-2013). The present study was approved by the ethics committee of the Hospital das Clínicas, University of São Paulo, Brazil (SDC: 3485/10/ 074), and each participant provided written informed consent before participation.

Based on this cohort study, 1225 individuals were selected following some inclusion and non-inclusion criteria. Initially, all the participants answered a questionnaire in which they stated whether they were affected by some type of diabetes or if they were users of some antidiabetic medication. If such questions had affirmative answers, the patient was not included in the study. After responding to the questionnaire, regardless of responses, patients were referred for blood screening. After screening test, T2DM was diagnosed by the presence of fasting plasma glucose $\geq 126 \mathrm{mg} / \mathrm{dL}$ or antidiabetic drug use [9]. In such case, the subject was not included in the cycle 1. Therefore, in cycle 1 we assessed only non-diabetic patients, accordingly to the criteria above (questionnaire and fasting plasma glucose). Five years after cycle 1, cycle 2 of the study was carried out.

In cycle 2 the patients in cycle 1 were reassessed and divided into two groups: (i) those who remained non-diabetic (Diabetes Free group) and (ii) those who had fasting plasma glucose levels greater than $126 \mathrm{mg} / \mathrm{dL}$ or reported use of antidiabetic drug (Incident Diabetes group).

In both cycles, blood triglycerides, total cholesterol, HDL-cholesterol, LDL-cholesterol and fasting glucose were evaluated by standard techniques in 12-h fasting blood samples [10].

Dyslipidaemia treatment was defined as percentage of individuals who used at least one class of lipid-lowering drug.

Anthropometric parameters were measured according to a standard protocol [10]. Height was measured in centimetres and weight in kilograms using a calibrated digital balance. WC was measured at the mean point between the lowest rib margin and the iliac crest with the subject standing and at the maximum point of normal expiration. Hip circumference was measured to the nearest $0.1 \mathrm{~cm}$ around the thighs, at the height of the greater trochanter, in the standing position. Increased WC was defined as $\geq 88 \mathrm{~cm}$ for women and $\geq 102 \mathrm{~cm}$ for men. The calculation of BAI was based on hip circumference and height $\left(\mathrm{BAI}=\left[\right.\right.$ hip circumference $(\mathrm{cm}) /\left(\right.$ height $\left.(\mathrm{m})^{1.5}\right]$ 18) [5].

Also in both cycles, blood pressure was measured using a standard digital sphygmomanometer (OMRON, Brazil) on the left arm after $5 \mathrm{~min}$ rest, in the sitting position. Systolic (SBP) and diastolic blood pressures (DBP) were calculated from three readings (mean value of all measurements), with a minimal interval of 3 min [10]. Hypertension was defined as mean $\mathrm{SBP} \geq 140 \mathrm{mmHg}$ and/or $\mathrm{DBP} \geq 90 \mathrm{mmHg}$ and/or antihypertensive drug use.

Clinical characteristics of patients in both cycles were assessed using descriptive statistics. Continuous variables were expressed as the mean $\pm \mathrm{SD}$ and categorical variables were expressed as percentages. Normality of all data was tested with the Kolmogorov-Smirnov test. Mixed-effects logistic regression was used in order to verify the association between BAI and the incidence of T2DM, and examine how much risk it represents for the development of this chronic disease. The regression model was carried out having BAI as the main parameter, and sex, age, SBP, triglycerides and HDL-cholesterol as control variables. Taking into account the kinship relations among patients, we also considered family as an effect. All statistical analyses were carried out using the $R$ (version 3.5.1) statistical software [11] with the level of significance set at $5 \%$.

\section{Results}

Clinical, demographic, anthropometric, and biochemical data are summarised in Table 1. The percentage of men, hypertensives, obese (increased WC) and individuals treated with lipid-lowering drugs were higher in the group that became diabetic after 5-year follow-up. In addition, these individuals showed higher SBP, BMI, WC and fasting glucose levels. BAI increased only $3 \%$ between cycles 1 and 2 . However, BAI was much higher in diabetics than non-diabetics in the cycle 2 (Fig. 1). In the present study, the incidence of T2DM after 5-year follow-up was $6.7 \%(75 / 1121)$.

Using mixed-effects logistic regression we found that BAI was significantly different between groups (p-value $<0.001$ ). The main finding of our study was that the elevation of a single unit of BAI represented an increase of $8.4 \%$ in the risk of patient developing T2DM (odds ratio $[\mathrm{OR}]=1.084[95 \%$ CI 1.045-1.124]) (Table 2). 
Table 1 Characteristics of subjects in the sample

\begin{tabular}{|c|c|c|c|c|}
\hline \multirow[t]{2}{*}{ Variables } & \multirow[t]{2}{*}{ Cycle 1} & \multicolumn{3}{|l|}{ Cycle 2} \\
\hline & & Diabetes free & Incident diabetes & $p$-value \\
\hline$n$ & 1121 & 1046 & 75 & - \\
\hline Age, years & $42.1 \pm 16.1$ & $46.9 \pm 15.9$ & $49.3 \pm 16.6$ & 0.24 \\
\hline Sex (\% men) & 44 & 43 & 47 & $<0.001$ \\
\hline Hypertension (\%) & 30 & 37 & 75 & $<0.001$ \\
\hline Increased WC (\%) & 30 & 42 & 61 & $<0.001$ \\
\hline Dyslipidaemia treatment (\%) & 3 & 7 & 24 & $<0.001$ \\
\hline $\mathrm{SBP}, \mathrm{mmHg}$ & $125.0 \pm 18.5$ & $125.2 \pm 16.4$ & $133.4 \pm 17.8$ & $<0.001$ \\
\hline $\mathrm{DBP}, \mathrm{mmHg}$ & $78.2 \pm 11.2$ & $76.7 \pm 10.6$ & $76.9 \pm 10.3$ & 0.88 \\
\hline $\mathrm{BMl}, \mathrm{kg} / \mathrm{m} 2$ & $24.3 \pm 4.7$ & $25.64 \pm 4.9$ & $28.45 \pm 5.9$ & $<0.001$ \\
\hline$W C, \mathrm{~cm}$ & $86.6 \pm 11.79$ & $90.6 \pm 11.8$ & $99.1 \pm 11.6$ & $<0.001$ \\
\hline Fasting glucose, mg/dL & $87.5 \pm 16.7$ & $89.3 \pm 10.2$ & $137.3 \pm 49.7$ & $<0.001$ \\
\hline Total cholesterol, mg/dL & $178.1 \pm 46.9$ & $200.6 \pm 67.2$ & $206.7 \pm 51.9$ & 0.33 \\
\hline HDL-cholesterol, mg/dL & $56.4 \pm 15.7$ & $47.5 \pm 11.8$ & $46.2 \pm 11.2$ & 0.33 \\
\hline LDL-cholesterol, mg/dL & $96.4 \pm 42.8$ & $125.3 \pm 34.8$ & $121.6 \pm 44.9$ & 0.51 \\
\hline Triglycerides, mg/dL & $128.9 \pm 68.9$ & $123.8 \pm 94.8$ & $115.7 \pm 78.6$ & 0.39 \\
\hline
\end{tabular}

Hypertension: systolic blood pressure $\geq 140 \mathrm{mmHg}$, diastolic blood pressure $\geq 90 \mathrm{mmHg}$ and/or anti-hypertensive drug use. Dyslipidaemia treatment: percentage of individuals who used at least one class of lipid-lowering drug. Increased WC: $\geq 88 \mathrm{~cm}$ for women and $\geq 102 \mathrm{~cm}$ for men. Continuous data are expressed as the mean \pm standard deviation and categorical data are expressed as percentage

SBP, systolic blood pressure; DBP, diastolic blood pressure; BAI, body adiposity index; BMI, body mass index; WC, waist circumference

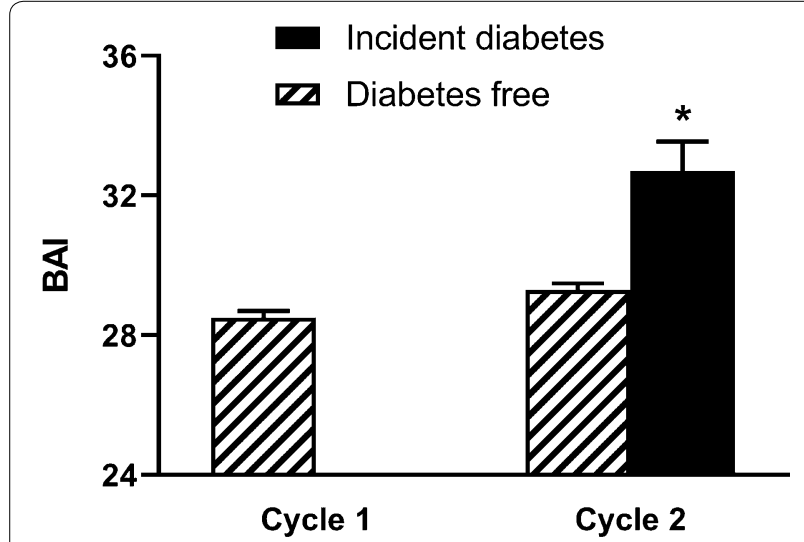

Fig. 1 Graph showing BAl in cycle 1 and in two groups of cycle 2. Data are mean and SEM. The increase of BAI in Incident Diabetes group was statistically significant $(p<0.001)$
This model was adjusted for SBP, sex, age, triglycerides and HDL-cholesterol.

\section{Discussion}

The main finding of our study was the association between BAI and T2DM in a Brazilian population in which the elevation of a single unit of BAI represented an increase of $8.4 \%$ in the risk of patient developing T2DM, even after adjusting for confounding variables such as age, sex, triglycerides, HDL-cholesterol and SBP.
Table 2 Variables associated with diabetes mellitus in a logistic regression analysis

\begin{tabular}{lllr}
\hline Variables & OR & $\mathbf{9 5 \%} \mathrm{Cl}$ & $\boldsymbol{p}$-value \\
\hline BAl & 1.084 & $1.045-1.124$ & $<0.001$ \\
Sex & 1.306 & $0.764-2.234$ & 0.329 \\
Age & 1.004 & $0.988-1.020$ & 0.633 \\
SBP & 1.025 & $1.011-1.040$ & $<0.001$ \\
Triglycerides & 1.000 & $0.997-1.003$ & 0.956 \\
HDL & 0.994 & $0.972-1.016$ & 0.597 \\
\hline
\end{tabular}

Diabetes mellitus: diagnosis was established in patients with fasting glucose equal to or greater than $126 \mathrm{mg} / \mathrm{dL}$, or in patients who were under the use of anti-diabetic medicine

Predictive variable: BAI

Control variables: sex, age, PAS, triglycerides and $\mathrm{HDL}$

$B A I$, body adiposity index; $S B P$, systolic blood pressure; $H D L$, high-density lipoprotein cholesterol

Several studies have focused on the relationship between anthropometric indices of adiposity and diabetes mellitus in samples from different populations. In Brazil, Flor et al. [3] and Freitas [12] demonstrated a strong association between T2DM and obesity, considering different Brazilian population samples. Supporting such studies, our findings confirm this relationship, highlighting BAI as an effective parameter. Corroborating with the findings of Bergman et al. [5] and López et al. [7], our study highlights that an increasing BAI implies 
a significant increase in the risk of developing T2DM. Therefore, BAI is a relevant tool to predict T2DM risk in the Baependi population.

This study has some limitations. First, all the participants live in Baependi, a small town with great rural activity located in South-eastern Brazil. Therefore, these results cannot be extrapolated to the general Brazilian population. Second, because it is an observational study where the participant makes a single visit per cycle, it becomes impracticable to diagnose diabetes through two measures of fasting glycaemia on different days. Therefore, like other observational studies, the diagnosis of diabetes is based on a single measurement of fasting glucose $(\geq 126 \mathrm{mg} / \mathrm{dL})$ or if the participant reports the use of hypoglycaemic drugs. On the other hand the main strengths are that our study has an appropriate sample size, which allowed for high statistical power, and, additionally, the anthropometric measures were obtained by a single investigator.

In conclusion, obesity is recognised as one of the most important risk factors for T2DM. The results of our study showed that BAI could be a useful tool for the assessment of T2DM risk in a Brazilian population.

\section{Abbreviations}

BMI: body mass index; BAI: body adiposity index; WC: waist circumference; OR: odds ratio; T2DM: type 2 diabetes mellitus; PAS: systolic blood pressure; DBP: diastolic blood pressure.

\section{Acknowledgements}

We are grateful to the population of Baependi for their participation in the Baependi Heart Study.

\section{Authors' contributions}

C.M.O, J.P and R.O.A participated in the design of the study, performed the statistical analysis and drafted the manuscript. C.A.M.J contributed to the acquisition and interpretation of the data. J.E.K contributed to the conception and design of the study. A.C.P conceived the study, participated in its design, and coordinated and assisted with the drafting of the manuscript. All authors read and approved the final manuscript.

\section{Funding}

The current research was supported by grants from São Paulo Research Foundation (FAPESP). This work was supported by Hospital Samaritano Society (Grant No. 25000.180.664/2011-35), through the Ministry of Health to Support Program Institutional Development of the Unified Health System (SUS-PROADI).

\section{Availability of supporting data}

The data sets used and/or analysed during the current study are available from the corresponding author on reasonable request.

\section{Ethics approval and consent to participate}

The study protocol was approved by the ethics committee of the Hospital das Clínicas (SDC: 3485/10/074), University of São Paulo, Brazil, and each subject provided informed written consent before participation.
Consent for publication

Not applicable.

\section{Competing interests}

The authors declare that they have no competing interests.

\begin{abstract}
Author details
${ }^{1}$ Laboratory of Genetics and Molecular Cardiology, Heart Institute (InCor), University of São Paulo Medical School, São Paulo, Brazil. ${ }^{2}$ Department of Integrative Medicine, Federal University of Paraná, Curitiba, Brazil. ${ }^{3}$ Department of Statistics, Pontificia Universidad Católica de Chile, Santiago, Chile. ${ }^{4}$ Department of Physiological Sciences, Federal University of Amazonas, Manaus, Brazil. ${ }^{5}$ Department of Physiology, Federal University of Juiz de Fora, Juiz de Fora, Brazil.
\end{abstract}

Received: 21 March 2019 Accepted: 17 July 2019

Published online: 29 August 2019

\section{References}

1. Malta DC, Felisbino-Mendes MS, Machado IE, de Azeredo Passos VM, de Abreu DMX, Ishitani LH, et al. Risk factors related to the global burden of disease in Brazil and its Federated Units. Rev Bras Epidemiol. 2015;20(1):217-32.

2. de Oliveira Alvim R, Mourao-Junior CA, de Oliveira CM, Krieger JE, Mill JG, Pereira AC. Body mass index, waist circumference, body adiposity index, and risk for type 2 diabetes in two populations in Brazil: General and Amerindian. PLoS ONE. 2014;9(6):e100223.

3. Flor LS, Campos MR. The prevalence of diabetes mellitus and its associated factors in the Brazilian adult population: evidence from a population-based survey. Rev Bras Epidemiol. 2017;20(1):16-29.

4. Lichtash CT, Cui J, Guo X, Chen YDI, Hsueh WA, Rotter Jl, et al. Body adiposity index versus body mass index and other anthropometric traits as correlates of cardiometabolic risk factors. PLoS ONE. 2013;8(6):e65954.

5. Bergman RN, Stefanovski D, Buchanan TA, Sumner AE, Reynolds JC, Sebring NG, et al. A better index of body adiposity. Obesity. 2011;19(5):1083-9.

6. Bennasar-Veny M, Lopez-Gonzalez AA, Tauler P, Cespedes ML, VicenteHerrero T, Yañez A, et al. Body adiposity index and cardiovascular health risk factors in Caucasians: a comparison with the body mass index and others. PLoS ONE. 2013;8(5):e63999.

7. López AA, Cespedes ML, Vicente T, Tomas M, Bennasar-Veny M, Tauler P, et al. Body adiposity index utilization in a Spanish Mediterranean population: Comparison with the body mass index. PLoS ONE. 2012;7(4):e35281.

8. Egan KJ, Schantz M, Negrão AB, Santos HC, Horimoto AR, Duarte NE, et al. Cohort profile: the Baependi Heart Study-a family-based, highly admixed cohort study in a rural Brazilian town. BMJ Open. 2016;6(10):e011598.

9. Padilha K, Venturini G, Pires TF, Horimoto ARVR, Malagrino PA, Gois TC, et al. Serum metabolomics profile of type 2 diabetes mellitus in a Brazilian rural population. Metabolomics. 2016;12:156.

10. Oliveira CM, Pereira AC, de Andrade M, Soler JM, Krieger JE. Heritability of cardiovascular risk factors in a Brazilian population: Baependi Heart Study. BMC Med Genet. 2008;9(32):1-8.

11. R Core Team. R: A language and environment for statistical computing. R Foundation for Statistical Computing, Vienna, Austria, 2019. https:// www.R-project.org.

12. de Freitas ICM, de Moraes SA. Social vulnerability effect over obesity anthropometric indexes: results from population-based epidemiological study. Rev Bras Epidemiol. 2016;19(2):433-50.

\section{Publisher's Note}

Springer Nature remains neutral with regard to jurisdictional claims in published maps and institutional affiliations. 